\title{
Flattening COVID-19 Curve in Egypt: An Epidemiological Modelling
}

\author{
Ali A. Hasab \\ Department of Epidemiology, High Institute of Public Health, Alexandria University
}

\begin{abstract}
Background: Flattening the curve refers to community isolation measures that keep the daily number of disease cases at a manageable level for medical providers. Efforts to completely contain the COVID - 19 have failed. As there is currently no vaccine or specific medication to treat COVID-19, the only way to flatten the curve is through collective action of mitigation efforts.

Objectives: The study was carried out with aim to study the effect of mitigation efforts in reducing the overall COVID -19 attack rate and flattening the curve of COVID-19 in Egypt. Methods: Secondary data in the situation reports of WHO, Worldometer and Egyptian MoHP Report about the COVID-19 epidemic in Egypt were analysed till April 24, 2020. The Susceptible Infected Recovered (SIR) model was used to study the epidemic dynamics of COVID-19 pandemic.

Results: In Egypt in fourth week after exposure $\beta$ was 0.27 and $\gamma=0.06$. The fraction of infectious individuals will grow exponentially as the exponential growth rate was 0.21 , with an epidemic time of 4 days. $\mathrm{R}$ in $23^{\mathrm{rd}}$ April it was 0.18 (less than one person infected) where $\beta=0.07$ and $\gamma$ $=0.39$. There was reduction in the actual overall attack rate up to 23 th April of $70 \%$ of its value without intervention. There was flattening of the curve.

Conclusion and recommendation: Mitigation efforts of Egypt had succeeded in flattening the curve of COVID-19 Pandemic. Even though, strengthening all the mitigation efforts to keep the exposure probability, as well as the transmission rate as low as possible is mandatory for containment of the epidemic.
\end{abstract}

Keywords: COVID-19, Egypt, Epidemic SIR Model, Pandemic, Flattening the curve

\section{Correspondence:}

Email: aliabdelhalim1948@gmail.com 


\section{Introduction}

The Coronavirus pandemic is the defining global health crisis of our time. Since its emergence in late 2019, the virus has spread to every continent. Countries are racing to slow the spread of the disease... Efforts to completely contain the COVID - 19 the pandemic responsible for infecting more than three and half millions with the disease, have failed. In less than two months, the global number of confirmed COVID-19 cases increases more than thirty times . That infection rate, scary as it sounds, hides just how much the out of control virus has spread, especially in the hardesthit. $(1,2)$

Flattening the curve refers to community isolation measures that keep the daily number of disease cases at a manageable level for medical providers In epidemiology, the idea of slowing a virus' spread so that fewer people need to seek treatment at any given time is known as "flattening the curve A flatter curve assumes the same number of people ultimately get infected, but over a longer period of time. A slower infection rate means a less stressed health care system, fewer hospital visits on any given day and fewer sick people being turned away. ${ }^{(3,4)}$

As there is currently no vaccine or specific medication to treat COVID-19, and because testing is so limited in many countries, the only way to flatten the curve is through collective action of mitigation efforts together with epidemiologic surveillance, contact tracing and epidemiological testing where the contacts of infected people are identified, tested in turn and isolated as needed. ${ }^{(5)}$

The study was carried out with aim to study the effect of mitigation efforts in reducing the overall COVID -19 attack rate and the consequential excess mortality attributed to the pandemic, delay and reduce the peak attack rate together with flattening the curve of COVID-19 pandemic in Egypt. 


\section{Methods}

The data was collected essentially by using secondary data. All cases and deaths in the situation reports of $\mathrm{WHO}^{(6)}$ and the Worldometer till April $24^{\text {th }}, 2020$ were included in the study. ${ }^{(7)}$ Every case was represented by a row. Death was coded as 1 and cases by 0 . Egyptian Ministry of Health $\&$ Population (MoHP) report issued on the $8^{\text {th }}$ of April, 2020, was also included ${ }^{(8)}$. The data were entered, analyzed by IBM SPSS Statistics, 25th edition ${ }^{(9)}$. CFR in this study was calculated as the number of deaths divided by the total cases with defined outcome (either died or cured). Wolfram Player 12 software was used for the Susceptible Infected Recovered (SIR) epidemic dynamics of COVID-19. (10)

The following SIR model was considered: where $\mathrm{S}$ is the fraction of susceptible individuals, $\mathrm{I}$ is the fraction of infectious individuals, and $\mathrm{R}$ is the fraction of recovered individuals, $\beta$ is the transmission rate per infectious individual, and $\gamma$ is the recovery rate. So in the SIR model, members of a population are categorized into one of three groups: those who are susceptible to being infected, those who have been infected and are able to spread the disease to susceptible individuals, and those who have recovered from the disease. Movement of individuals is one-way only, S - I - R.

\section{Results}

In Egypt In the fourth week after exposure ( $3^{\text {rd }}$ to $9^{\text {th }}$ March 2020) the number of reported cases was 59 and the recovered 4 . The recovery rate was calculated by dividing the recovered cases by the reported cases, $4 / 59=0.06$. $\beta$ was calculated by the equation:

$\mathrm{C}=\beta \mathrm{SI}-\mathrm{I} \gamma$

$166=\beta \times 102332404 \times 59-59 \times 0.06$

$\beta=0.27$ 
Where $\mathrm{C}$ is the incidence of cases in the second week, $\mathrm{S}$ is the susceptible population, $\mathrm{I}$ is the number of cases in fourth week after exposure and $\gamma$ is the recovery rate

Note that the basic reproduction number is $R=\beta / \gamma$ the basic reproductive rate $(R)=4.5$ (four and half person infected). If transmission rate - recovery rate $<0$, the fraction of infectious individuals will not grows exponentially and the incidence rate (number of new cases per unit time) also will not increase exponentially. In Egypt in fourth week after exposure $\beta$ was 0.27 and $\gamma=0.06$. The fraction of infectious individuals will grow exponentially and the exponential growth rate will be $\lambda=\beta-\gamma$. i.e. $0.27-0.06=0.21$, during the early phase of the epidemic time was about 4 day $(\operatorname{In} 2 /$ $\lambda)$

Calculating $\mathrm{R}$ in $23^{\text {rd }}$ April it was 0.18 (less than one person infected) where $\beta=0.07$ and $\gamma=0.39$.

Studying the distribution of actual and projected cases without intervention by week since exposure, Table (1) shows the exponential growth of the cases. The actual overall attack rate up to $23^{\text {th }}$ April was 40 per million and the projected rate without intervention was 145 . This difference is significant $(\mathrm{cOR}=3.625(2.554-5.145), \mathrm{P}=0.0000)$ with $73 \%$ reduction of its value without intervention. As for deaths, it was projected bases on $25 \%$ of deaths with defined outcome in Egypt ${ }^{(11)}$. Figure (1) shows how the epidemic cure was flattened in Egypt. The same picture was observed for deaths (Figure 2)

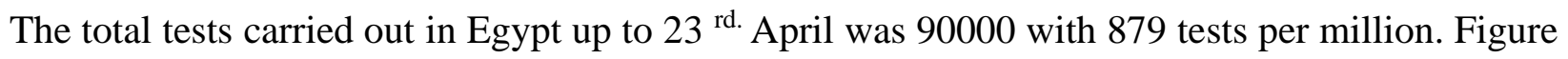
(3) shows that there was no significant correlation between the number of tests and CFR (deaths with defined outcome) by country $(\mathrm{r}=0.08, \mathrm{P}=0.947)$. The bed occupancy rate in the quarantine centers was $60 \%$ at MoHP report, Egypt issued at April 8. Based on $\beta$ of 0.5 and $\gamma$ of 0.2 of the projected cases at $23^{\text {rd }}$ of April, figure (4) shows the reduced fatalities from COVID-19 Pandemic in Egypt at $23^{\text {rd }}$ April 2020. Figure (5A, B\&C) shows the SIR Epidemic Dynamics of COVID-19 in the early stage of the epidemic and that of the projected and actual cases at $23^{\text {rd. April. }}$

Table (1) Distribution of actual and projected cases and deaths by week since exposure in Egypt (3 ${ }^{\text {rd }}$ March-23 ${ }^{\text {rd }}$ April) 


\begin{tabular}{|l|c|c|c|c|}
\hline Week & Actual cases & Projected cases & Actual deaths & Projected deaths \\
& & & & \\
\hline 4.00 & 59.00 & 59.00 & & \\
\hline 5.00 & 166.00 & 166.00 & 6.00 & $\mathbf{6 . 0 0}$ \\
\hline 6.00 & 232.00 & 581.00 & 15.00 & $\mathbf{2 9 0 . 5 0}$ \\
\hline 7.00 & 323.00 & 1162.00 & $\mathbf{3 1 . 0 0}$ & $\mathbf{5 8 1 . 0 0}$ \\
\hline $\mathbf{8 . 0 0}$ & $\mathbf{7 8 0 . 0 0}$ & $\mathbf{2 3 2 4 . 0 0}$ & $\mathbf{5 1 . 0 0}$ & $\mathbf{1 1 6 1 . 1 3}$ \\
\hline $\mathbf{9 . 0 0}$ & $\mathbf{9 4 5 . 0 0}$ & $\mathbf{4 6 4 4 . 5 0}$ & $\mathbf{8 0 . 0 0}$ & $\mathbf{2 3 2 0 . 5 0}$ \\
\hline 10.00 & 1140.00 & $\mathbf{9 2 8 2 . 0 0}$ & $\mathbf{8 5 . 0 0}$ & $\mathbf{5 0 0 . 0 0}$ \\
\hline 11.00 & 1500.00 & $\mathbf{1 8 5 6 4 . 0 0}$ & $\mathbf{9 0 . 0 0}$ & \\
\hline
\end{tabular}

Figure ( 1 ) Distribution of COVID-19 actual and projected cases without intervention by week since exposure in Egypt $\left(3^{\text {th }}\right.$ March $2^{\text {th }}$ April 2020)

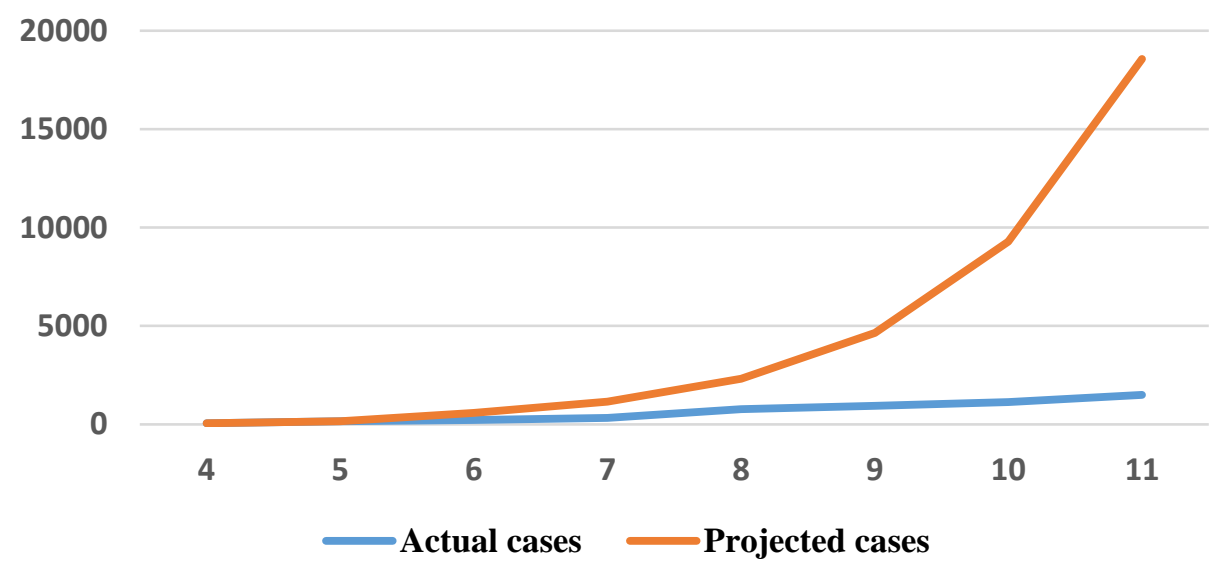


Figure ( 2 ) Distribution of COVID-19 actual and projected deaths without intervention by week since exposure in Egypt (10 ${ }^{\text {th }}$ March $-23^{\text {th }}$ April 2020)

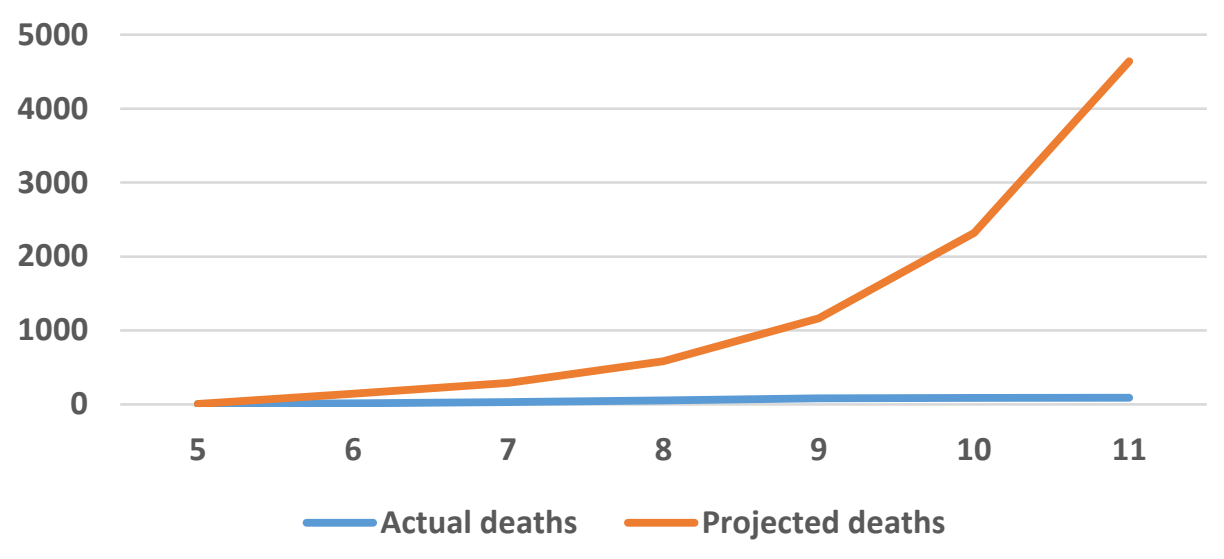

Figure ( 3 ) Distribution of RT-PCR and CFR by country, 23 ${ }^{\text {th }}$ April 2020

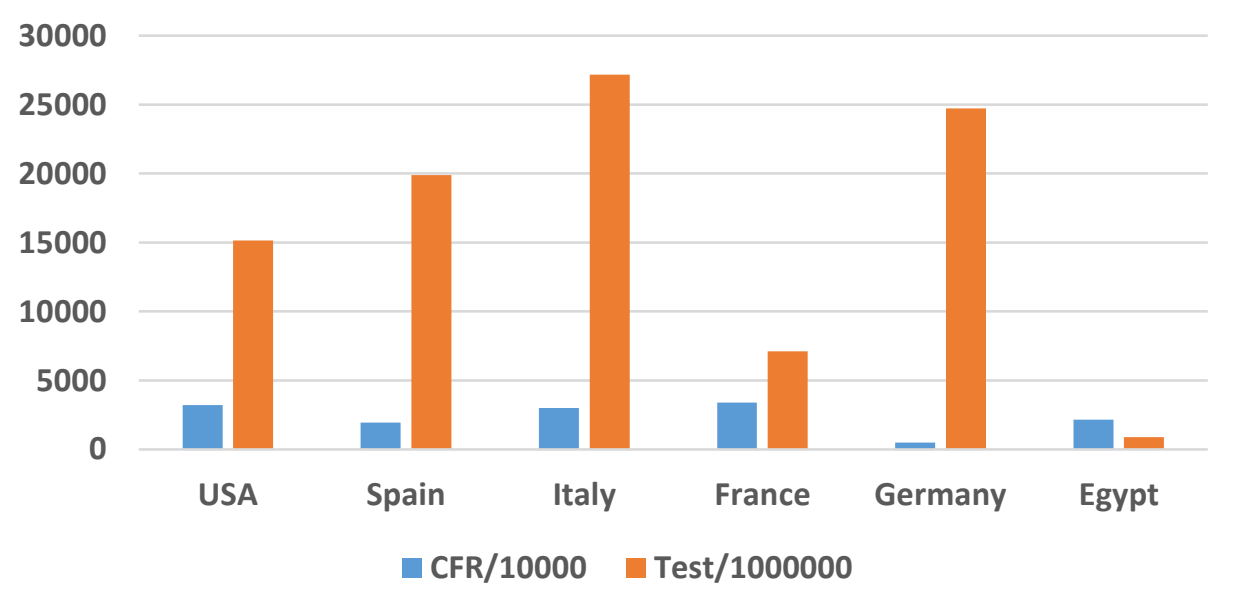




\section{Reducing Fatalities from Coronavirus Epidemic}

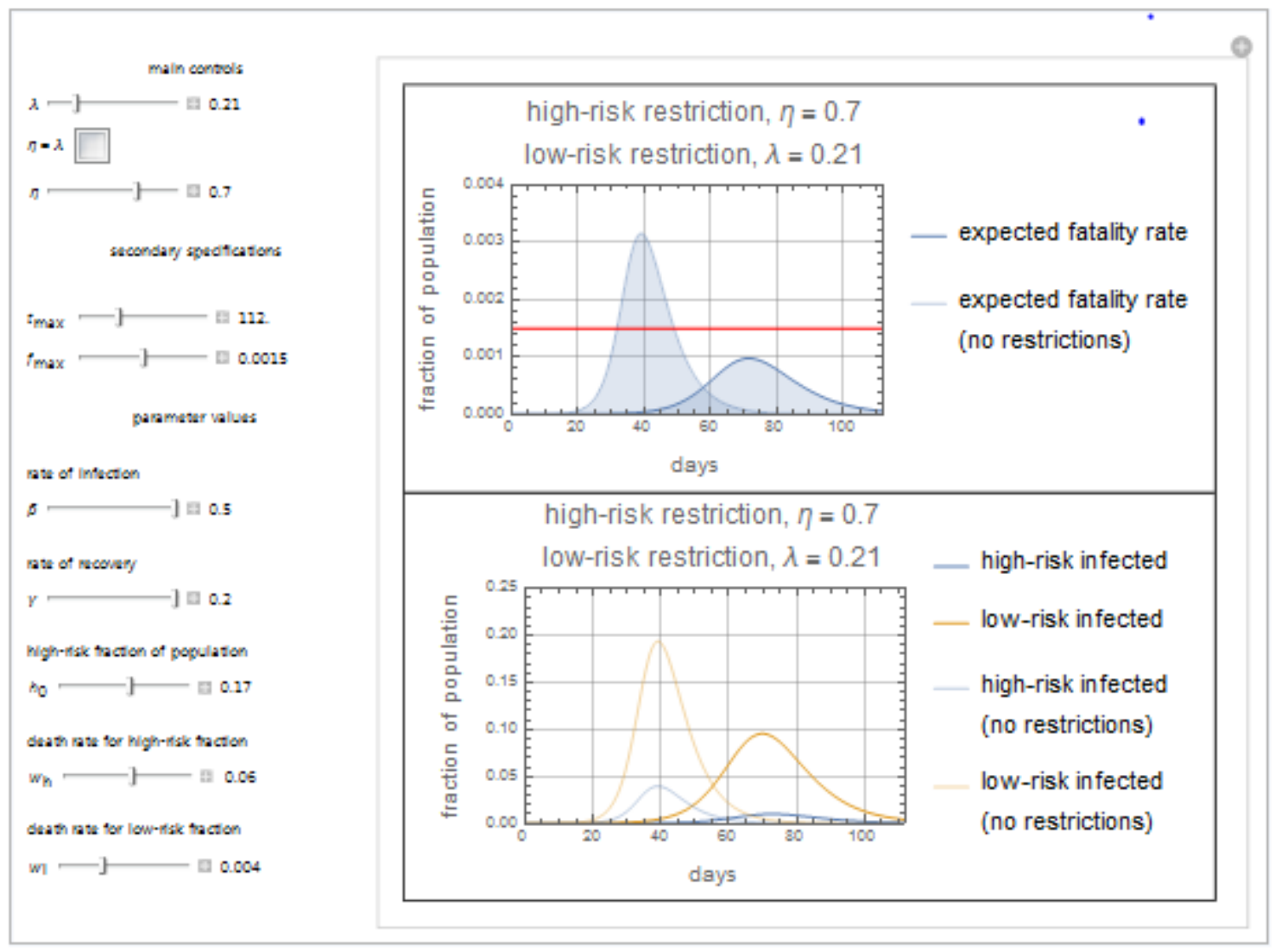

Figure (4) Reducing fatalities from COVID-19 Pandemic in Egypt at 23rd April 2020 


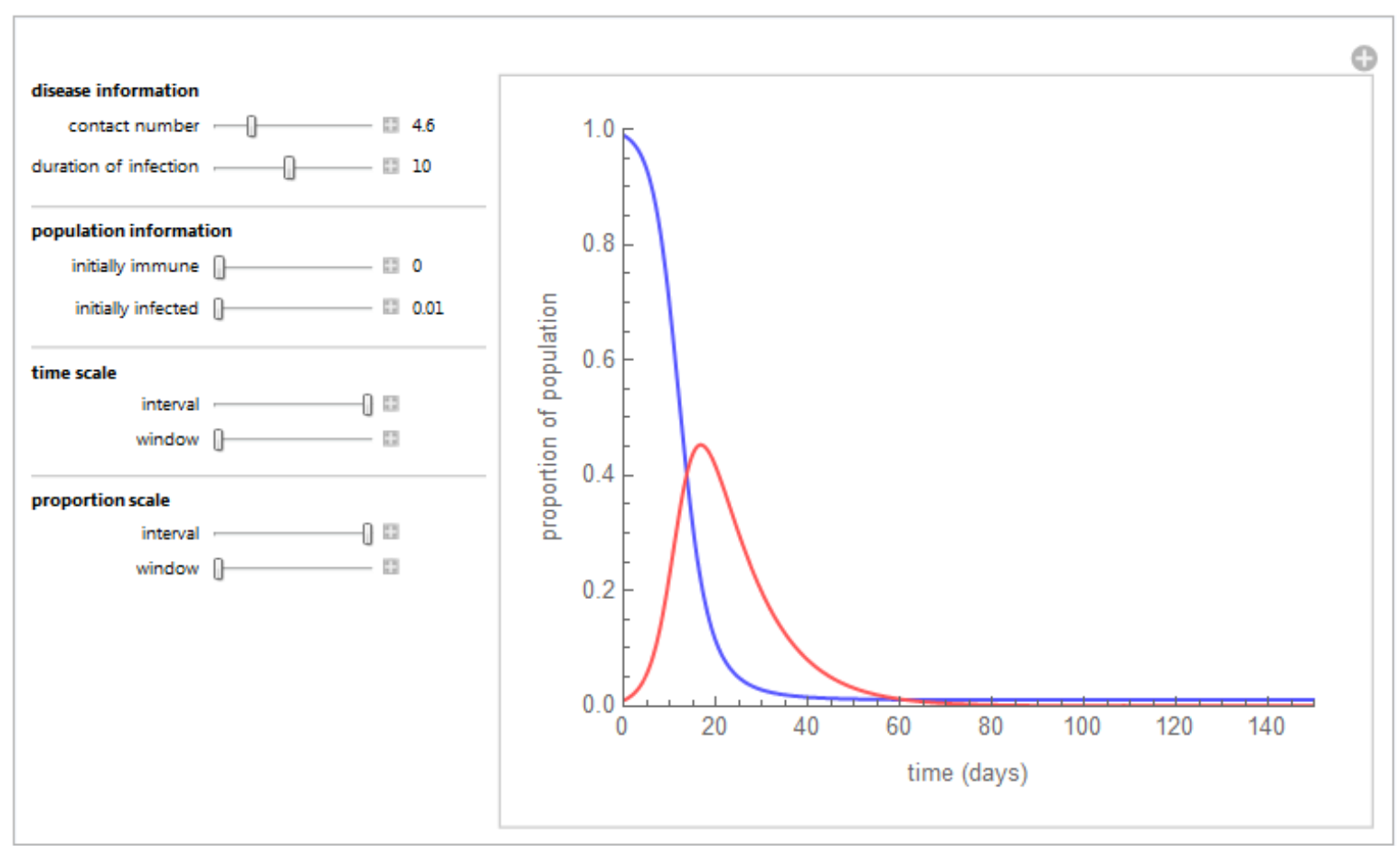

Figure (5A) SIR Epidemic Dynamics of COVID-19 at $3^{\text {rd }}$ March 2020 


\section{SIR Epidemic Dynamics}

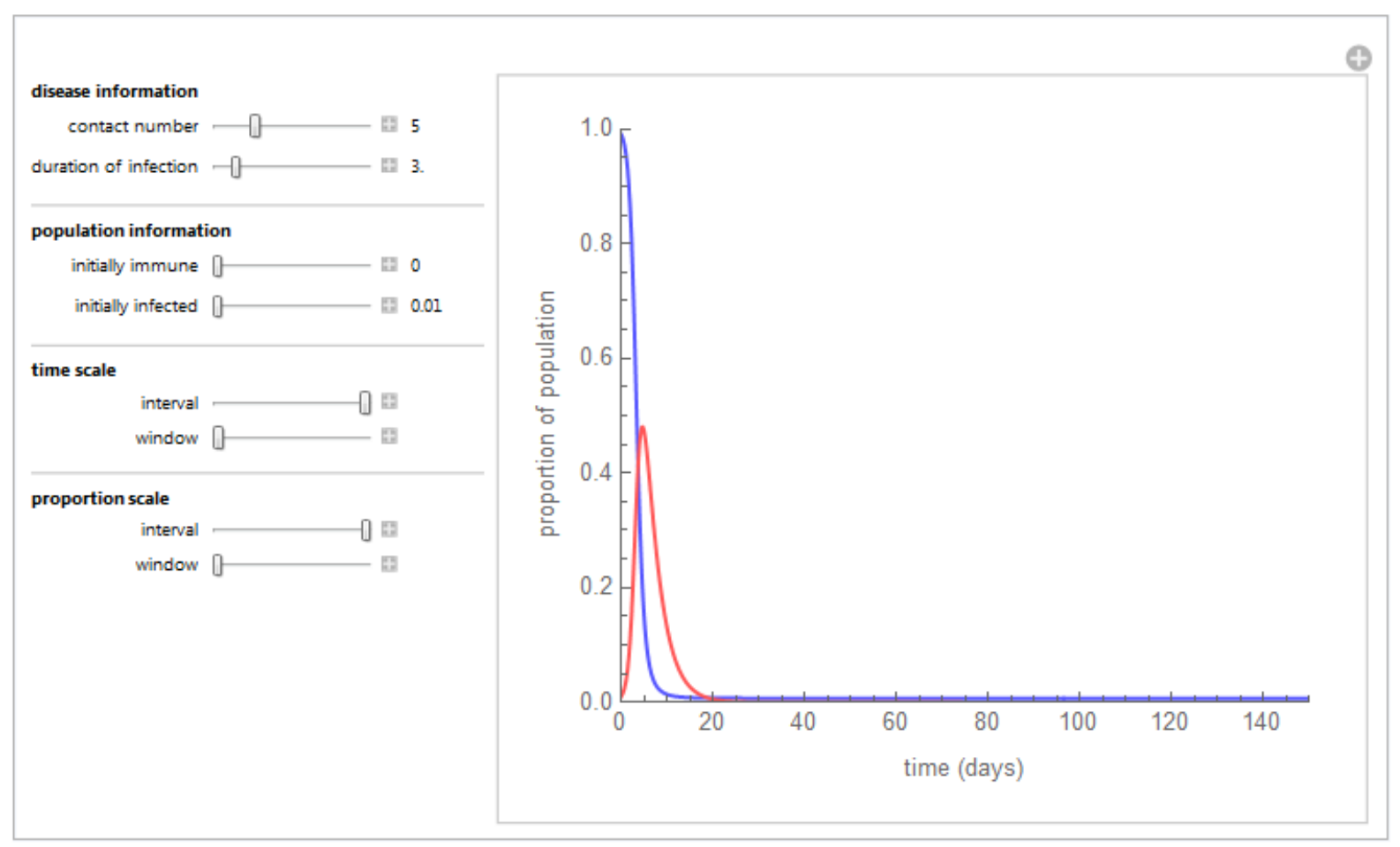

Figure (5B) SIR Epidemic Dynamics of Projected COVID-19 in Egypt at 23 April 2020 without intervention 


\section{SIR Epidemic Dynamics}

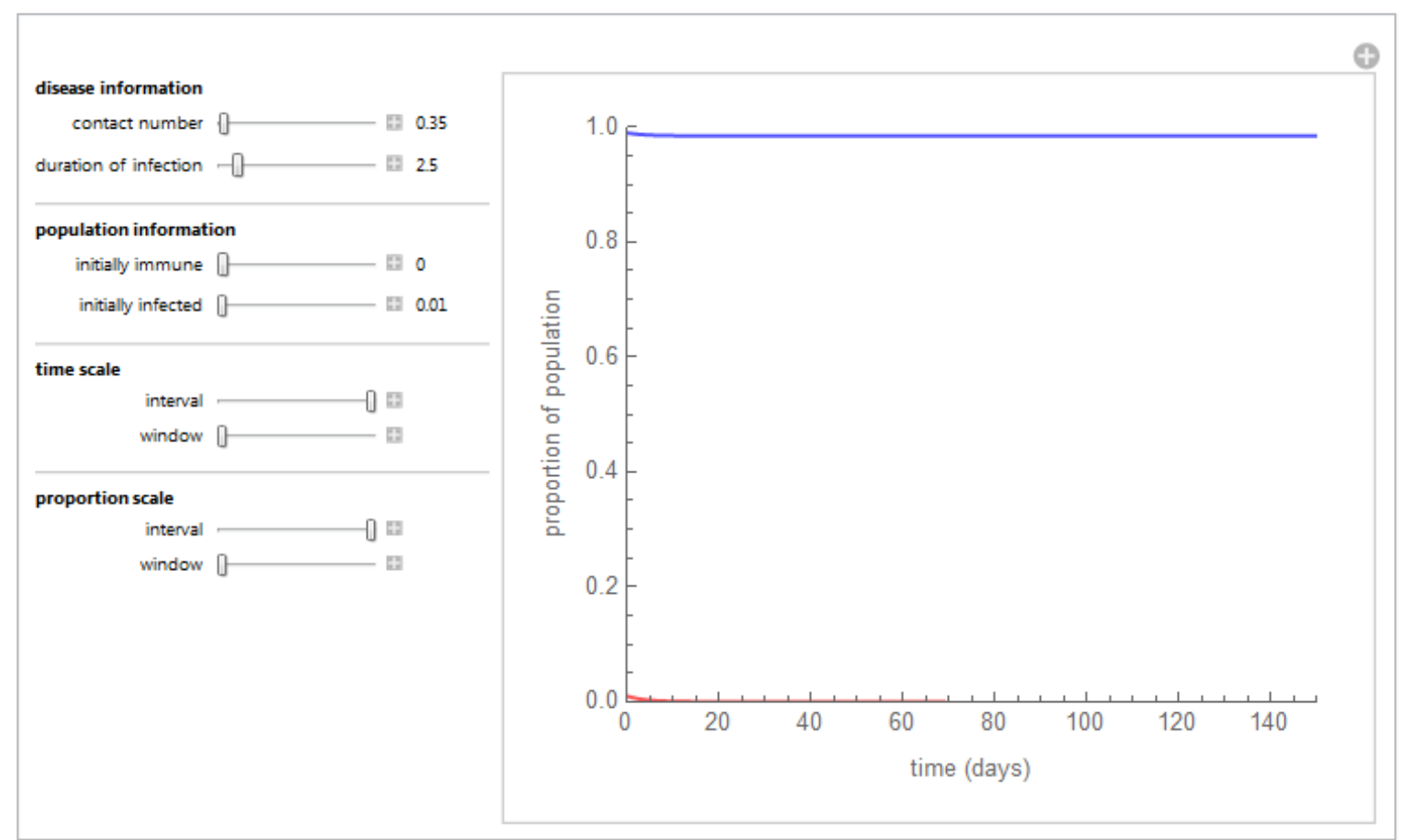

Figure (5C) SIR Epidemic Dynamics of Projected COVID-19 in Egypt at 23 April 2020 


\section{Discussion}

The situation of COVID-19 pandemic in Egypt as compared to other parts of the world is promising. Mitigation efforts of Egypt had succeeded in delaying the peak of the curve after the seventh week of first exposure. The transmission rate is smaller than the recovery rate with no exponential growth. Egypt is one of the countries, alongside China ${ }^{(11)}$, to flatten the curve of new infections of COVID-19 pandemic. Egypt has done so without huge outbreaks, without China's draconian restrictions on speech and movement, or economically damaging lockdowns like those in Europe and the United States. (11)

Much rather be ahead of the curve than behind it. To explain, Egypt did not underestimate the problem since the first reported case in Egypt on 8th February, knowing well that COVID-19 is inevitable and started to deal early, to limit transmission and increase recovery. Our results suggest a critical role of social distancing in the potential control of COVID-19 pandemic and indicate that such interventions are capable of arresting COVID-19 epidemic development, but only if they are used in combination, activated without delay and maintained for a relatively long period. Limiting transmission in Egypt was difficult without the mitigation procedures of the government as the household and social contact is high (three contacts, as the household number is 4 in Egypt). ${ }^{(12,13)}$ WHO COVID-19 technical mission in Egypt on March, 5 concluded that considerable efforts have been made by the government in allocating additional dedicated human and financial resources needed to contain the outbreak...(14) The mission also added that Egypt's strong disease surveillance system and contact tracing efforts have proven effective in controlling and managing sporadic and clusters of cases before they can spread. Epidemiological testing — where the contacts of infected people are identified, tested in turn and isolated as needed — is the only way to fully break the chains of transmission, says. Without it, the virus will come roaring back as soon as social distancing guidelines are relaxed. Recent border closures now provide an opportunity to enhance screening and rapid testing capacity using a risk assessment approach. However it is important to note the sensitivity of RT-PCR testing, is less than optimal. The area under the cure of RT-PCR is about $75 \%$ with a fair discrimination power. ${ }^{(15)}$ A single negative RT-PCR should not exclude 
COVID-19, especially if clinical suspicion is high. The combination of clinical symptoms, exposure history, typical CT imaging features, and dynamic changes must be considered to identify COVID-19 with higher sensitivity. This explains the non-significant correlation of case fatality rate observed in this study by the tests carried out.

Besides, significant efforts have been made to ensure the public is updated on the current situation, including working with other sectors to reach vulnerable populations. Greater coordination and partnerships with civil society, non-governmental organizations (NGOs), and the media that ensure that the public can make informed decisions, without succumbing to mistrust and the stigma associated with the disease ${ }^{(16,17)}$ However, as epidemiologists, we need to be very cautious with our optimism taking into account those who are COVID-19 positive, but have not been tested and the number of people with either mild illness or who are non-symptomatic.

Conclusion and recommendation: Mitigation efforts of Egypt had succeeded in flattening the curve of COVID-19 Pandemic. Even though, strengthening all the mitigation efforts to keep the exposure probability, as well as the transmission rate as low as possible is mandatory for containment of the epidemic. More studies is needed to evaluate the validity of the RT-PCR as a predictor of COVID-19 is needed.

\section{REFERENCES}

1. Wang C, Horby PW, Hayden FG, Gao GF. A novel coronavirus outbreak of global health concern. Lancet. 2020; 395(10223):470-3. doi: 10.1016/S0140-6736(20)30185-9.

2. WHO. Coronavirus disease 2019 (COVID-19): situation report. Geneva: World Health Organization, 2020107.

3. Brandon Specktor. Coronavirus: What is 'flattening the curve,' and will it work? 16 March 2020

4. Wikipedia. Flatten the cure https://en.wikipedia.org/wiki/Flatten the curve

5. Editorial Board NY times. The Epic Failure of Coronavirus Testing in America. China and South Korea offer lessons in how to curb this pandemic.

6. WHO. Coronavirus disease 2019 (COVID-19): situation report. Geneva: World Health Organization, 202095.

7. Worldmeter. COVID-19 coronavirus pandemic. United States: Dadax; 2008 [updated 2020; cited 2020 April 10]. Available from: https://www.worldometers.info/ coronavirus/.

8. MOHP Report of COVID-19, Egypt, 2nd, April, 8 Final. Egyptian MOHP: 2020.

9. IBM Corp. Released 2017. IBM SPSS Statistics for Windows VA, NY: IBM Corp. 
10. Mathematica [Internet]. Wolfram Research, Inc. Available from: https://www.wolfram.com/mathematica.

11. Hasab A, El-Ghitany E, Ahmed N. Situational Analysis and Epidemic Modeling of COVID-19 in Egypt .JHIPH 2020;50(1):46-51

12. Zhu ZB, Zhong CK, Zhang KX, Dong C, Peng H, Xu T, et al. [Epidemic trend of corona virus disease 2019 (COVID-19) in mainland China]. Zhonghua Yu Fang Yi Xue Za Zhi. 2020; 54(0):E022.

13. Egypt's efforts to contain coronavirus are 'reassuring': Sisi. Ahram online. 2020 Tuesday 31 Mar 2020.

14. STATISTICA. Number of households in Egypt from 2012 to 2017 [cited 2020 April, 2]. Available from: https://www.statista.com/statistics/875743/egyptl-number-ofhouseholds/.

15. Hasab A. Validity of RT-PCR as predictor of COVID-19. Unpublished data.2020

16. WHO-EMRO. WHO delegation concludes COVID-19 technical mission to Egypt [Internet]. 2020; 25 March 2020 [cited April, 5]. Available from:

http://www.emro.who.int/media/news/ who-delegation-concludes-covid-19-technicalmission-to-egypt.html

17. Al-Mandhari A, Samhouri D, Abubakar A, Brennan R. Coronavirus Disease 2019 outbreak: preparedness and readiness of countries in the Eastern Mediterranean Region. East Mediterr Health J. 2020; 26(2):136-7. 